P499 SINGLE-FIBRE EMG ANALYSIS OF THE MATURATION OF THE NEUROMUSCULAR ENDPLATE DURING REINNERVATION AFTER TRAUMATIC NERVE INJURY

Philip Broser*. Kinderspital, Sankt Gallen, Switzerland

10.1136/archdischild-2019-epa.835

Nerve injuries are a common complication of upper extremities fractures. Especially the long arm nerves are prone to injuries. After such an injury the muscles are denervated. Fortunately, if nerves are injured but the continuity of the nerve shed is intact a regeneration by spreading of the axonal fibres starts early after the injury and finally a new neuromuscular connection - a neuromuscular endplate- is reestablished. Till today it is unknown whether the newly formed neuro-muscular endplate is functional equivalent to the original. During the last years a new technic became routinely available in paediatric neurology to study the neuro-muscular endplate: stimulated single-fibre-EMG. This technic allows a precise and objective assessment of the neuro-muscular connection. At our hospital we care together with our colleagues from the paediatric surgery department for children with fractures and nerve injuries. During the last years we have adopted the technique of single fibre EMG and included it into our clinical repertoire for traumatic nerve injuries.

It has been previously hypothesis that the newly formed neuromuscular endplate is not as reliable as the original one. This fact would have major clinical implications for the support and training after nerval injuries. To address this question, we have set up this pilot project to conduct routine measurements of the recovering nerve using single fibre-EMG. Our preliminary data shows, that these measurements can be reliable conducted in the pediatric population and we are now in the process of applying for ethical approval to analyse a larger cohort.

\section{P500 SANDIFER SYNDROME: PRESENTATION, INVESTIGATION AND MANAGEMENT}

Irina Mindlina*. University of Cambridge, Cambridge, UK

10.1136/archdischild-2019-epa.836

Background Sandifer syndrome is a rare complication of gastro-oesophageal reflux disease (GORD) when a patient presents with extraoesophageal symptoms, typically neurological. Although its pathophysiology is not completely understood, one explanation is that neurological manifestations are the consequence of vagal reflex with the reflex centre in nucleus tractus solitarii. The goal of this study was to review the existing literature and describe a typical presentation and most appropriate investigations and management for the Sandifer syndrome.

Methods A comprehensive literature search was performed via PubMed, Cochrane Library and NHS Evidence databases. 27 cases and observational studies were identified.

Results The literature demonstrates that presenting symptoms of Sandifer's may include any combination of abnormal movements and/or positioning of head, neck, trunk and upper limbs, seizure-like episodes, ocular symptoms, irritability, developmental and growth delay, iron-deficiency anaemia.
In terms of diagnostic procedures, 24-hour oesophageal $\mathrm{pH}$ monitoring was positive in all the cases of Sandifer's where it was performed, while upper GI endoscopy \pm biopsy and barium swallow were diagnostic only in a subset of cases.

A range of treatment options were applied in the reviewed literature, including dietary changes, pharmacological management, enteral tube feeding, and surgical approach. These treatment options are consistent with the 2015 NICE guidelines on management of GORD in children and young people.

Successful treatment of the underlying gastro-oesophageal pathology led to a complete or near-complete resolution of the neurological symptoms in all of the cases.

Discussion As Sandifer syndrome is driven by the underlying gastro-oesophageal reflux, it is not surprising that its investigations and management showed to be consistent with those of GORD. It is evident from the literature that many patients were originally misdiagnosed with various neuropsychiatric diagnoses that led to unnecessary testing and ineffective medications with significant side effects. Earlier diagnosis of Sandifer's would have allowed to avoid them.

\section{P501 FUNCTIONAL HEMISPHEROTOMY AS A TREATMENT OF CHOICE IN REFRACTORY RASMUSSEN SYNDROME}

Bojan Marjanovic*, Vladimir Pavlovic. Primary Health Center, Lazarevac, Serbia

10.1136/archdischild-2019-epa.837

Rasmussen syndrome is a subacute inflammatory encephalitis and one of the causes of continual partial seizures. The onset of convulsive attacks is usually preceded by an episode of non-specific fever, usually of viral origin (eg, CMV infection). Attacks are usually partial, long-lasting and frequent. It most often occurs in children under 10 years of age. EEG findings show diffuse paroxysmal activity. The disease is progressive, with development of various neurological deficits (hemiplegia, hemianopsia, and aphasia). Here we present a case of a 3 year-old girl, with normal early psychomotor development, who had the first absence-type convulsive attack during her holidays in Greece in 2016. Treatment with valproic acid was initiated. In the next two months, two more seizures followed, accompanied with fever (eyes fixation, arm and leg swings, squinting, non-response to calls for 10 seconds). Both times she was hospitalized, and during the second hospitalization, EEG showed the existence of generalized epileptiform changes. MR of the endocranium was normal. Due to poor control of attacks and their frequency, new antiepileptics (lamiktal, carbamazepine, topamax) have been introduced into the therapy. In March 2017, new MR of the endocranium showed the excistence of a hypotrophy of the left hemisphere and descreet left-sided Walerian degeneration, when diagnosis of Rasmussen's syndrome was suspected. Further diagnostic procedures included immunological examination of the liquor and the presence of oligoclonal IgG strains in the liquor (LGI1, AMPA, GABA-B) was confirmed. Anti-NMDA autoantibodies were negative, and therapy with specific immunoglobulins was administered. Corticosteroids were also administered during the exacerbations. In a further course of the disease, a clinical finding reveals a worsening of neurological status (spastic right-sided hemiparesis, facial nerve paralysis and dysarthria). At the end of 2017 , the girl had an average of $4-5$ convulsive 
attacks per day. With medical therapy, physical and speech therapies were regularly performed. Because of progression of the disease and poor response to therapy, surgical treatment was proposed. In September 2018, left functional hemispherotomy was performed at the clinic of neursciences in Paris, after which the convulsions completely disappeared. From all of the above, we conclude that functional hemispherotomy is a therapy of choice in the treatment of resistant attacks in Rasmussen's syndrome, which stops progression of the disease and prevents further neurological damage.

\section{P502 YERSINIA ENTEROLITICA INFECTION WITH ENCEPHALOPATHY}

laurent Pradeaux*, Nabil Bendjazia, marie Percot-Blondy, loic Fritsch, maud Sordet, sandra Pochelu, daniela Behrendt. Centre Hospitalier, Périgueux, France

10.1136/archdischild-2019-epa.838

We want to describe a 8 year old boy with no medical problem before. The boy came at the emergency department at the night with general weakness, hallucinations and difficulties to walk.

When he arrived the temperature was $37,1^{\circ}$, glycemia 6,6 $\mathrm{mmol} / \mathrm{l}$. He had normal vital signs, a pulse rate of 95 beats per minute and a normal blood pressure $(107 / 53 \mathrm{mmHg}$ ). $\mathrm{He}$ was lethargic, had hallucinations and he had a cerebellar ataxia. Pupils were dilated but reactive. Reflexes were normal. On admission standard blood test (cell count, hepatic parameter, C-reactive protein) was normal. Urine and serum toxicology screen was negative. Cerebral fluid examination revealed no white cell and a normal glucose, protein concentration. The electroencephalogram showed left temporal slow waves. Brain magnetic resonance imaging (MRI) was normal. Herpes simplex virus encephalitis was suspected and aciclovir was started. Three days later the aciclovir was stopped (PCR herpes negative), PCR enterovirus as well.

At the stool culture, we found a yersinia enterocolitica, biotype 4, serotype 0:3. This pathogen agent was the only one we found. We began an antibiotic treatment when we got the result but the boy's clinical state improved before with no more ataxia at day two of hospitalisation. The hallucinations persisted during four days. The boy was discharged at day eight. Four weeks later the electroencephalogram and MRI were normal. Clinical examination was perfect.

Yersinia enterolitica is a common cause of acute gastroenteritis in our countries. The contamination by fecal-oral route, invasive forms (abscesses, septicemia) occurs in patients with underlying conditions (cirrhosis, diabetes). Secondary complications such as reactive arthritis or erythema nodosum are known. An acute or chronic neurological disease is possible but rare.

On a follow up of 458 hospitalized patients in Norway 14 presented neurological manifestations. The different expressions were headache, dizziness, polyradiculitis. Another report of an 8 years old girl appeared similar to our present case (1). Yersinia enterolitica is not know to secrete a neurotoxin. Yersinia enterolitica secrete a heat stable enterotoxin and can induce immunologic response. Mechanism of acute or chronic neurological manifestations remain unclear but must not be disregard these and could induce a field of search (2).

\section{REFERENCES}

1. Berner R, Kist M, Sauer M. Encephalopathy associated with yersinia enterolitica. The Lancet 1998; $351: 418$

2. Bancerz-kisiel A, Pieczywek M, Lada P, Szweda W. The most important virulence markers of yersinia enterolitica and the role during infection. Genes $2018 ; 9$ :235-249.

\section{P503 AN EYE-OPENING CASE ANISCORIA}

${ }^{1,2}$ Elinor Jenkins*, ${ }^{2,3}$ David W Webb, ${ }^{2}$ Mary 0 Regan. ${ }^{1}$ Trinity College Dublin, School of Medicine, Dublin , Ireland; ' ${ }^{2}$ Our Lady's Hospital for Sick Children , Dublin , Ireland; ${ }^{3}$ Trinity College Dublin, School of Medicine, Dublin, Ireland

\subsection{6/archdischild-2019-epa.839}

Introduction Aniscoria is a condition characterised by pupils of unequal size. Aniscoria may be completely benign and physiological. However it can also be associated with serious lifethreatening conditions and therefore prompts alarm in clinicians.

Case Description A 4-year-old boy referred by his family doctor to hospital with a sudden onset dilated and un-reactive left pupil.

His mother had noticed the abnormal pupil on waking that morning, approximately 6 hours earlier. Her son did not appear to be in pain and his vision was unaffected. He appeared to be rubbing his eye at times but his awareness and behaviour were unaltered. There was no history of trauma and he was systemically well.

Background This little boy had a known diagnosis of Creatine Transporter Deficiency, an inherited metabolic disorder associated with learning disability and epilepsy. Physically he was mobile and very active with a mild torticollis, recurrent epistaxis and a tendency to salivate excessively. There was no recent history of headache, nausea or change in seizures. Medications at the time of presentation included Epilim Chrono (450 mg nocte), Flixotide $(125 \mathrm{mcg})$ and a Scopoderm patch.

Examination confirmed a fixed dilated left pupil with a left esotropia but otherwise normal eye movement, no ptosis and no obvious conjunctival injection. His neurological exam revealed no altered awareness and no motor deficit.

Discussion Aniscoria is common - found in approximately $20 \%$ of children (Fierz, F et al 2017). However the asymmetry is mild and pupil reactive. Aniscoria of $>1.3 \mathrm{~mm}$ is unlikely to be physiologic in children and warrants careful assessment (Suh et al 2016).

In the absence of a third nerve lesion or trauma to the eye, application of a pharmacological agent with mydriatic effect is the most likely explanation in childhood. Scopoderm patches have been reported as causing aniscoria in a 30-yearold nurse (Shah et al, 2017) and a 14-year-old boy (Lin, 2001). A recent article explored the use of hyoscine patches to reduce drooling in children with neurodisability (Parr et al, 2017) citing aniscoria as one of a number of side-effects when compared to glycopyrronium liquid.

Conclusion Following discussion with his mother involvement of his scopoderm patch was considered to be the most likely cause of his dilated pupil and no further investigations were felt necessary. His mydriasis fully resolved over three days. Children with a neuro-disability can be extremely active -making it hard to keep medication out of their way! 